\title{
Negative Muon Count for the FNAL Muon g-2 Experiment
}

\author{
By: Elettra Preosti ${ }^{a}$, Diktys Stratakis ${ }^{b}$ \\ ${ }^{a}$ University of California, Berkeley Department of Physics, Berkeley, CA, USA 94720 \\ ${ }^{b}$ Fermi National Accelerator Laboratory, Batavia, IL, USA 60510
}

\begin{abstract}
:
The primary goal of the FNAL Muon g-2 Experiment, located in Batavia, IL, USA, is to measure the anomalous magnetic dipole moment, $a_{\mu}=(g-2) / 2$, of a muon to a precision of $0.14 \mathrm{ppm}$. Initial results from the experiment confirm a discrepancy between the experimental value of the muon anomaly compared with the theoretical value first discovered by the Brookhaven Muon g-2 Experiment in 2001. This discrepancy hints at new physics. However, running the experiment with negative muons is essential in order to verify the experimental value of the muon anomaly. As neither experiments nor simulation studies pertaining to the muon anomaly have been performed with negative muons, the goal of this work is to estimate the rates of negative muons arriving at the FNAL Muon g-2 experiment so that scientists can estimate the time required to run the experiment with negative muons. In order to do this, we performed several simulations of the Muon Campus, which delivers muons to the FNAL Muon g-2 Experiment, using the G4Beamline v. 3.06 at the National Energy Research Computing Center. We performed separate simulations for different sections of the muon campus, and simulations were repeated twice, once for negative muons and once for positive muons. An initial $10^{9}$ protons were shot on target for both the negative and positive muon simulations. We found that the rate of negative muons arriving at the experiment was 0.549 times the rate of positive muons arriving at the experiment.
\end{abstract}

\section{INTRODUCTION:}

\subsection{Background:}

Muon g-2, located at Fermi National Accelerator Laboratory (FNAL) in Batavia, IL, USA, is a particle physics experiment that seeks to measure the anomalous magnetic dipole moment, $a_{\mu}=$ $(g-2) / 2$, of a muon to a precision of $0.14 \mathrm{ppm}$ [5]. The equation for $a_{\mu}$ can be derived from the equation for the magnetic moment of electrons and muons represented as follows:

$$
\mu=g \frac{q}{2 m} S
$$

where $\mu$ is defined to be the spin magnetic moment of a Dirac particle, $g$ is defined to be the gyromagnetic ratio, $q$ is defined to be the charge of the particle, $m$ is defined to be the mass of the particle, and $S$ is defined to be the value of the spin angular momentum [2]. 
The anomalous magnetic dipole moment of electrons and muons have been essential to the development of the Standard Model (SM) with the SM predicting the value for the muon anomaly to be $a_{\mu}=116491810(43) \times 10^{-11}(0.37 \mathrm{ppm})$ [5]. However, in 2001, the Brookhaven Muon g-2 Experiment concluded with $0.544 \mathrm{ppm}$ precision that the experimental value for the muon anomaly was 3.7 standard deviations higher than the theoretical value [2].

As this discrepancy hints at the possibility of new physics, The FNAL Muon g-2 Experiment hopes to confirm the results obtained by the Brookhaven Muon g-2 Experiment, but with higher precision. In fact, recently, in April 2021, the FNAL Muon g-2 Experiment released initial results with $0.46 \mathrm{ppm}$ precision indicating that there is indeed a discrepancy between the experimental and theoretical values of $a_{\mu}$; the combined results from FNAL and Brookhaven measured the experimental value for the muon anomaly to be $0.00116592061(41)$, which is 4.2 standard deviations higher than the theoretical value. Although this is just short of the 5 standard deviations required to claim a scientific discovery, these results could indicate that the SM is in need of revision. Moreover, the probability that the results are a statistical fluctuation is only 1 in $40,000[1]$.

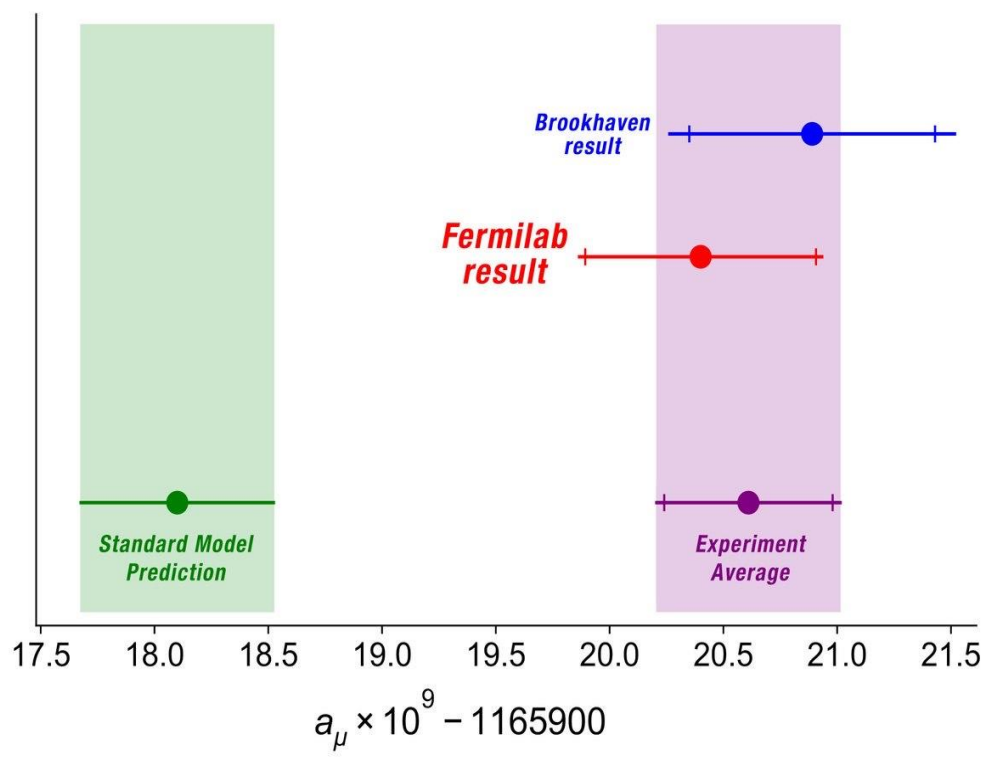

Fig 1: Results from the FNAL Muon g-2 Experiment as well as the Brookhaven Muon g-2 experiment compared with the SM prediction of the anomalous magnetic moment of muons.

\subsection{Motivation:}

As of now, all experiments as well as all simulation studies measuring $g$ have only used positive muons. However, it is essential to verify the measurement of $g$ with negative muons (i.e. to confirm that the same value of $g$ is obtained for both positive and negative muons). Otherwise, 
this could indicate yet another discrepancy in the SM. Moreover, running with negative muons could provide insight into the validity of Charge Parity Theory (CPT) as well as Lorentz Invariants.

Thus, the FNAL Muon g-2 experiment is planning to run with negative muons beginning in 2022. The goal of this study is to determine the rates of negative muons arriving at the experiment compared to the rates of positive muons arriving at the experiment. The results of this investigation will provide helpful information in estimating the required time the FNAL Muon g-2 experiment will need to run to measure $g$ using negative muons. In addition, the results presented in this simulation study will be the first ever presented involving negative muons.

\section{MUON CAMPUS:}

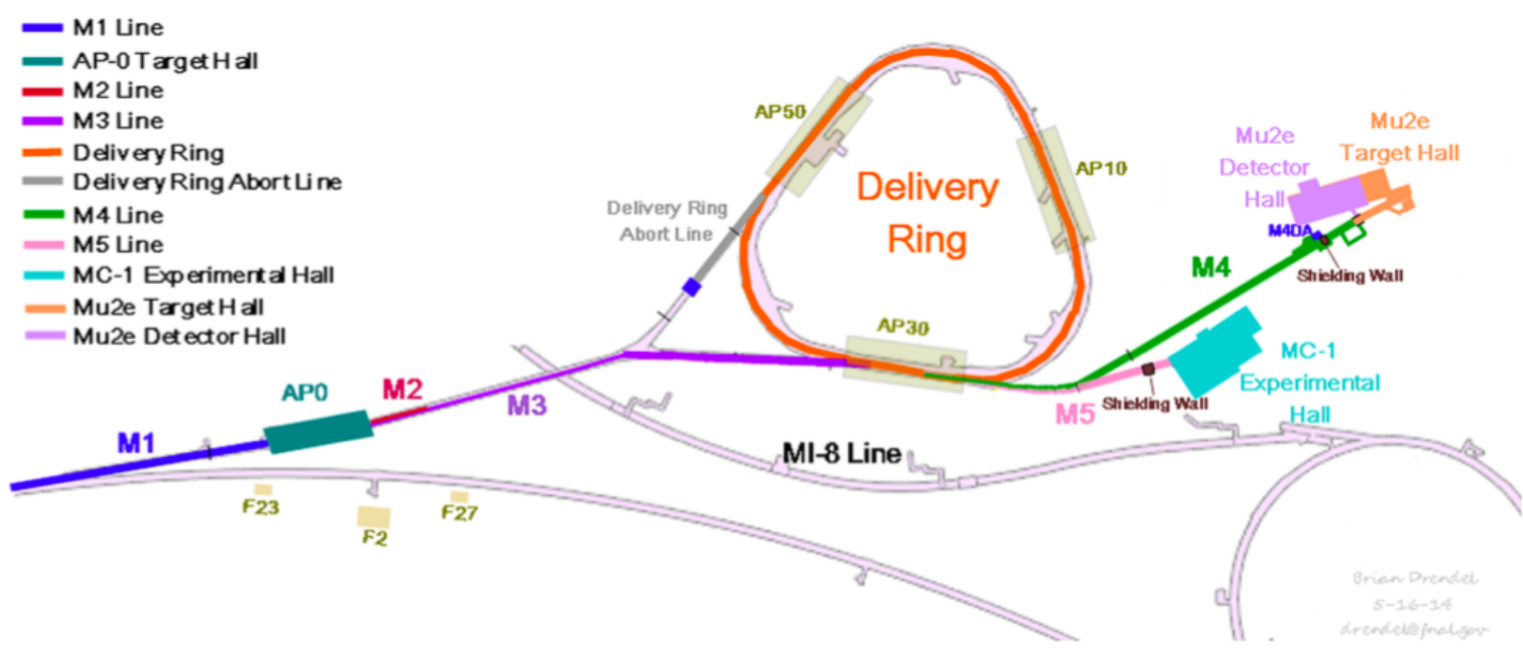

Fig 2: A schematic representation of the Muon Campus at Fermilab

The Muon Campus is the system through which muons are delivered to the FNAL Muon g-2 experiment, which must be run using muons having momentum within $\sim .2 \%$ of $3.094 \mathrm{GeV} / \mathrm{c}$. First, $8 \mathrm{GeV} / \mathrm{c}$ protons are delivered to an Inconel target in the AP-0 Target Hall through the M1 beamline. During the Muon g-2 experiment, 16 pulses of protons are sent to the Inconel target with a frequency of $12 \mathrm{~Hz}$. After striking the Inconel target, a secondary beam consisting primarily of protons, pions, kaons, muons, positrons, and deuterons is produced. The positive secondary particles are then focused by a lithium lens and moment selected via a pulsed dipole magnet (PMAG), which selects $3.094 \mathrm{GeV} / \mathrm{c}$ positive particles before bending them to the M2 beamline [4]. In this study, we will also simulate the scenario in which negative secondary particles are focused and moment selected by the lithium lens and the PMAG respectively. 
The secondary particles then travel through the M2 and M3 beamlines, which are designed to capture as many $3.094 \mathrm{GeV} / \mathrm{c}$ muons resulting from pion (and kaon) decays as possible. The secondary beam is then vertically injected into the Delivery Ring (DR) with a bend produced by a combination of a $\mathrm{C}$ magnet (CMAG), followed by a large-aperture focusing quad (Q303) and a pulsed magnetic septum dipole. In the DR, the muons are longitudinally separated from the protons before the protons are removed using a kicker. A separate kicker extracts the muons into the M4 beamline. The resulting muon beam is eventually transferred to the M5 beamline which leads to the FNAL Muon g-2 experiment Storage Ring (SR) where measurements for the experiment take place [4].

\section{METHODS:}

Simulations from this investigation were performed using the Geant4Beamline (g4bl) v. 3.06 program at the National Energy Research Scientific Computing Center (NERSC). In order to keep track of the negative to positive muon ratio throughout the simulation process, sections of the Muon Campus Accelerator were simulated separately beginning with a Gaussian proton beam just upstream of the AP-0 Target Hall. The sections were as follows: the AP-0 Target Hall ending at the lithium lens, the AP-0 Target Hall beginning just before the PMAG and entering the M2/M3 beamline including one turn in the DR, three additional turns in the DR (each simulated separately), and the M4/M5 beamlines. The output of each preceding section was used as the input for the following section, and the final negative to positive muon ratio was calculated from the output of the M4/M5 beamline.

A total of $10^{9}$ protons were sent to the target, and simulations were repeated twice, once for positive muons and once for negative muons.

\section{RESULTS:}

\subsection{AP-0 Target Hall:}

The target station consists of five main devices: a pion production target, a lithium lens, a copper collimator, a PMAG, and a beam dump. The pion production target comprises a cylindrical core made from a nickel-iron alloy called Inconel-600 encased by a thick layer of beryllium. Next, the magnetic focusing lithium lens, located $0.3 \mathrm{~m}$ downstream of the target, is made from a lithium rod with radius $0.01 \mathrm{~m}$ and length $0.16 \mathrm{~m}$ surrounded by a titanium encasing. Finally, the PMAG, which selects $3.094 \mathrm{GeV} / \mathrm{c}$ particles and bends them $3^{\circ}$ into the M2-line, is $1.03 \mathrm{~m}$ long and has a magnetic field strength of $0.53 \mathrm{~T}$. It is located $1.3 \mathrm{~m}$ downstream from the target [3].

Overall, the primary proton beam first interacts with the pion production target in order to produce a beam of secondary particles which are then focused by the lithium lens and momentum selected via PMAG. In order to perform simulations for negative muons, we reversed the polarity of the lithium lens and the PMAG as the original tracking program was written to keep only positive particles. 
We first examined the distribution of particles just downstream of the lithium lens where we found the ratio of negative to positive pions to be 0.523 and the ratio of negative to positive muons to be 0.449 . Furthermore, the ratio of negative to positive pions having momentum with $\sim 2 \%$ of $3.094 \mathrm{GeV} / \mathrm{c}$ was calculated to be 0.614 and the ratio of negative to positive muons having momentum with $\sim 2 \%$ of $3.094 \mathrm{GeV} / \mathrm{c}$ was calculated to be 0.375 . The table in Fig 3 . provides negative to positive pion ratios for additional momentums as well. However, because g4bl has been optimized for particles with energies of around $3.094 \mathrm{GeV} / \mathrm{c}$, the ratios of negative to positive pions at higher energies may not be representative of experimental results. The negative particle count was obtained from simulations performed using the modified tracking program where we reversed the polarity of the lithium lens whereas the positive particle count was obtained from simulations performed using the original tracking program. At this stage, both distributions contain both positive and negative particles. However, the distribution obtained from the modified tracking program contained predominantly negative particles whereas the distribution obtained from the original tracking program contained predominantly positive particles. Moreover, both distributions consisted mostly of pions and contained fewer kaons and muons.

Next, we examined the distribution of particles just downstream from the PMAG. Performing the same analysis as for the initial distribution, we found that the ratio of negative to positive pions to be 0.595 and the ratio of negative to positive muons to be 0.538 . Furthermore, the ratio of negative to positive pions having momentum with $\sim 2 \%$ of $3.094 \mathrm{GeV} / \mathrm{c}$ was calculated to be 0.570 and the ratio of negative to positive muons having momentum with $\sim 2 \%$ of $3.094 \mathrm{GeV} / \mathrm{c}$ was calculated to be 0.400 . As the PMAG has selected particles with $3.094 \mathrm{GeV} / \mathrm{momentum}$, the ratios obtained above are approximately the same. Again, both distributions contained both negative and positive particles and mostly contained pions.

\begin{tabular}{|c|c|}
\hline Momentum(GeV/c) $\pm 2 \%$ & $\pi$-/ $\pi+$ Ratio \\
\hline 1 & 0.480 \\
\hline 2 & 0.485 \\
\hline 3.094 & 0.615 \\
\hline 4 & 0.754 \\
\hline 5 & 1.559 \\
\hline 6 & 1.409 \\
\hline
\end{tabular}


Fig 3: The table above lists the negative to positive pion ratios at various momentums just after the lithium lens and prior to being moment selected by the PMAG. As g4bl has been optimized for particles with momentum 3.094 GeV/c, results at higher energies may not be representative of true experimental results.

\subsection{M2/M3 Beamlines}

The length of the combined M2 and M3 beamlines is $280 \mathrm{~m}$. Their purpose is not only to deliver muons to the DR, but also to capture as many muons having momentum within $\sim 2 \%$ of 3.094 $\mathrm{GeV} / \mathrm{c}$ that have decayed from pions and kaons as possible [3]. The M2 and M3 beamlines contain a series of bends and quads with the M2 beamline starting with four matching quadrupoles. When running simulations for negative particles, in order to capture as many negative muons as possible, we reversed the polarities of all of the bends and quads within the M2 and M3 beamlines. This includes CMAG, where we analyzed the distribution of particles shortly before they entered the DR.
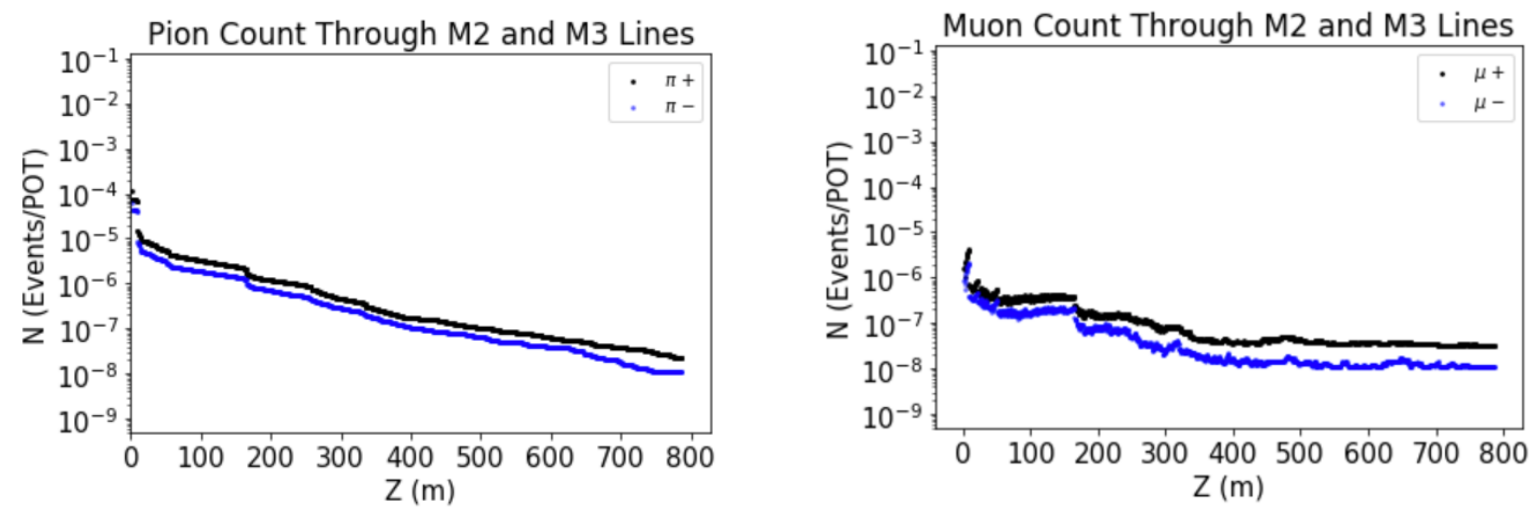

Fig 4: (Left) Positive and negative pion counts through the M2 and M3 beamlines including one turn in the DR. (Right) Positive and negative muon counts through the M2 and M3 beamlines including one turn in the DR.

Through the M2 and M3 beamlines, we see that the number of pions and muons decrease significantly both for the positive and negative muon simulations. Moreover, at the end of the M3 beamline, many of the pions have decayed to muons, although there are still significantly more pions than muons in both simulations. However the ratio of negative muons (resulting from the deck with reversed polarities) to positive muons (resulting from the original deck) is still approximately one half. The ratio of positive to negative particles only fluctuates slightly within the M2 and M3 beamlines. 


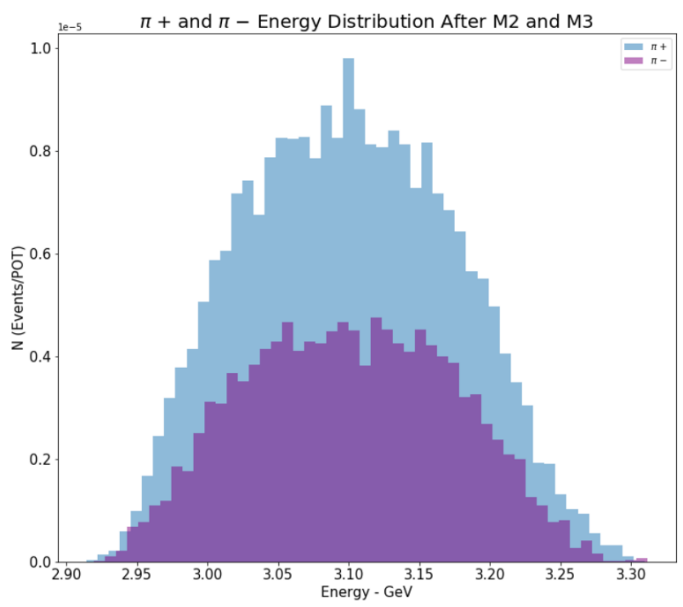

Fig 5: Positive and negative pion distributions at CMAG located towards the end of the M3 beamline.

\subsection{Delivery Ring}

At the end of the M3 beamline, the beam of secondary particles enters the DR, where it takes four turns. After four turns in the DR, the pions and kaons fully decay to muons, and the muons are then separated from any protons left in the secondary beam of particles. A kicker extracts the muons from the DR and inserts them into the M4 and M5 beamlines [4]. In our simulations, the first turn in the DR was included in the M2 and M3 beamlines simulation deck. The next three turns in the DR were each simulated separately.

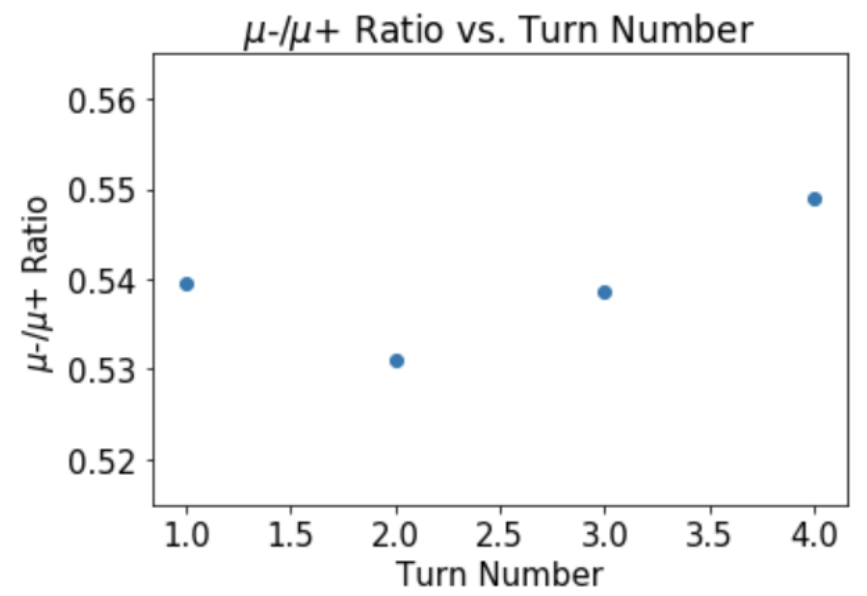

Fig 6: Negative to positive muon ratio after each turn in the DR.

From our results, we see that at the end of four turns in the Delivery Ring, the distribution of particles for both the positive and negative simulations consisted of muons, and there were no pions remaining after the second turn in the DR. In addition, in the simulation where the 
polarities of the lithium lens, PMAG, and all other bends, quads, and magnets were reversed to filter out positive particles, there were not any protons left in the distribution of particles. This could suggest that when running with negative muons, fewer turns in the DR are needed as muons will not need to be longitudinally separated from protons.

It is also important to note that the ratio of negative to positive muons having momentum within $\sim 2 \%$ of $3.094 \mathrm{GeV} / \mathrm{c}$ remains approximately half, fluctuating slightly with each turn in the DR. From the distribution of negative and positive muons, we also see that although most of the muons have momentum around $3.094 \mathrm{GeV} / \mathrm{c}$, some have a momentum that is significantly less than $3.094 \mathrm{GeV} / \mathrm{c}$. This is because some of the pions and kaons that decay to muons result in muons with energy less than $3.094 \mathrm{GeV}$.

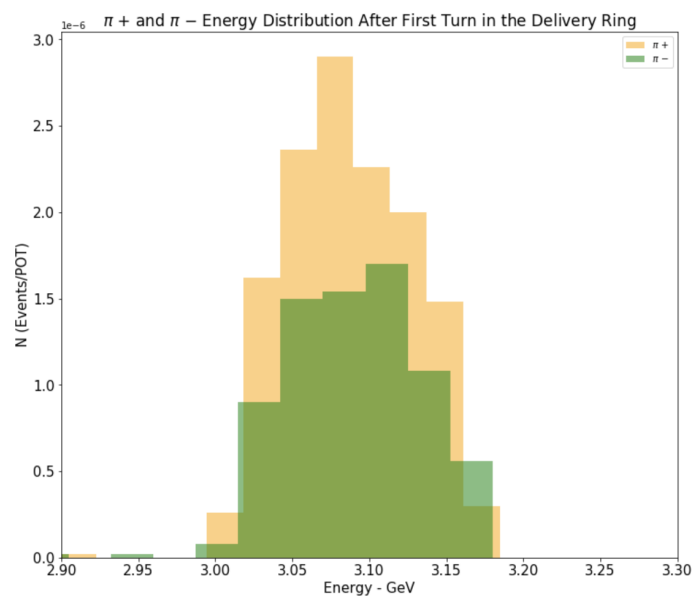

Fig 7: Positive and negative pion distributions after the first turn in the DR.

\subsection{M4/M5 Beamlines}

The M4 and M5 beamlines then take the clean muon beam extracted from the DR to the SR of the FNAL muon g-2 experiment. Our simulations begin at the start of the M4 beamline and end just before the muon beam enters the SR. The resulting distributions indicate that very few muons are lost in the M4 and M5 beamlines and that the ratio of negative to positive muons remains approximately constant, only fluctuating slightly. The final ratio of negative to positive muons is 0.549 , indicating that the rates of negative muons arriving at the SR will be just slightly over half the rate of positive muons. 
FN-1133-STUDENT

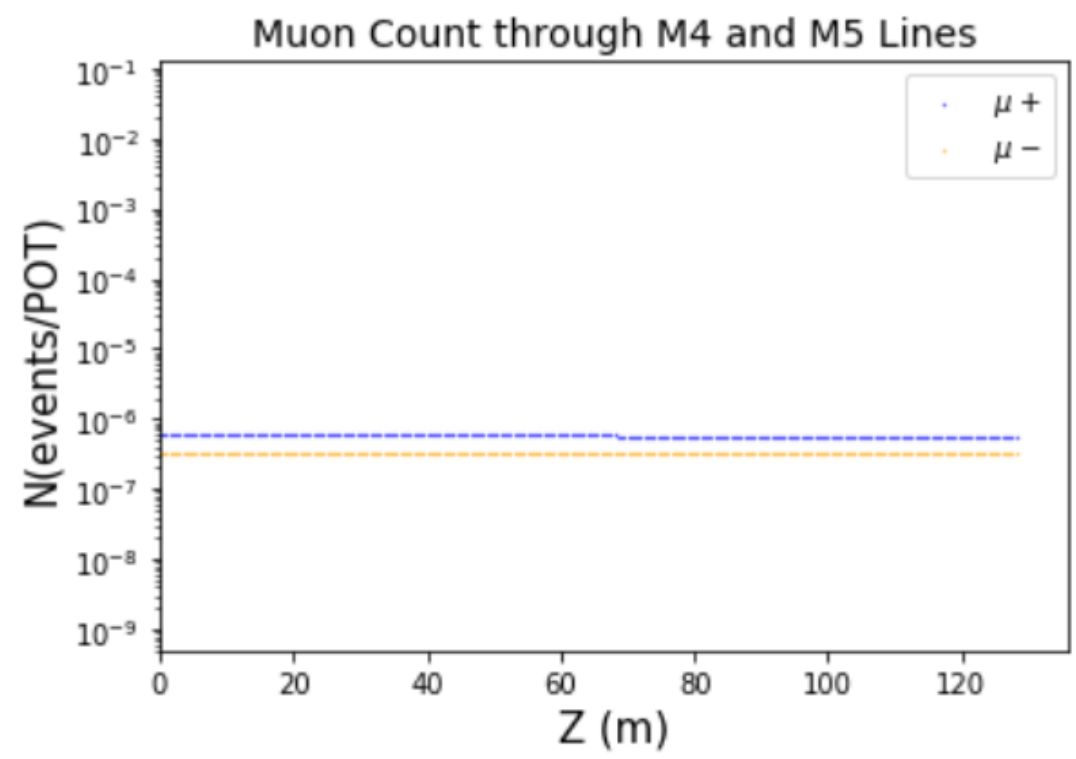

Fig 8: Positive and negative muon count through the M4 and M5 beamlines leading up the FNAL Muon g-2 Experiment

5. SUMMARY

\begin{tabular}{|c|c|c|}
\hline $\begin{array}{c}\text { Muon Campus } \\
\text { Location }\end{array}$ & $\boldsymbol{\pi}-/ \boldsymbol{\pi}+$ Ratio & $\boldsymbol{\mu}-/ \boldsymbol{\mu}+$ Ratio \\
\hline Lithium Lens (Post) & 0.614 & 0.375 \\
\hline PMAG (Post) & 0.570 & 0.400 \\
\hline CMAG & 0.532 & 0.507 \\
\hline DR (Turn 1) & 0.545 & 0.540 \\
\hline DR (Turn 2) & 0.529 & 0.531 \\
\hline DR (Turn 3) & - & 0.539 \\
\hline DR (Turn 4) & - & 0.549 \\
\hline SR Entrance & - & 0.549 \\
\hline
\end{tabular}

Fig 9: The table to the left indicates the ratio of positive to negative pions and positive to negative muons that have momentum within $2 \%$ of $3.094 \mathrm{GeV} / \mathrm{c}$ at different stages of the muon campus accelerator. There are no pions by Turn 3 in the DR. 
In summary, we found that the ratio of negative to positive muons arriving at the FNAL Muon g-2 Experiment is 0.549 . In fact, this ratio fluctuates only slightly at different locations of the Muon Campus. So, we can expect that by reversing all of the polarities of the components within the Muon Campus, given the same amount of running time, we can produce about half the number of negative muons as positive muons. However, since this simulation study only considered $10^{9}$ protons on target (POT), it resulted in approximately 300 negative muons and 600 positive muons at the end of the M5 beamline. Thus, for future studies, we recommend increasing the number of POTs by at least one order of magnitude. Moreover, it is important to repeat the simulations with other programs to confirm the results obtained from g4bl as g4bl is optimized only for particles around $3.094 \mathrm{GeV} / \mathrm{c}$.

\section{ACKNOWLEDGEMENTS :}

Thank you to Diktys Stratakis for his mentorship and guidance throughout this project. This work is supported by Fermi Research Alliance, LLC under Contract No. DE-AC02-07CH11359 with the United States Department of Energy.

\section{REFERENCES:}

1. Abi, B., et al. (2021). Measurement of the Positive Muon anomalous magnetic moment to 0.46 ppm. Physical Review Letters, 126(14)

2. Albahri, T. (2021), et al. Magnetic-field measurement and analysis for the Muon $g-2$ Experiment at Fermilab. Phys. Rev. A 103, 042208

3. Stratakis, D., et al. (2017). Accelerator performance analysis of the FERMILAB Muon Campus. Physical Review Accelerators and Beams, 20(11). https://doi.org/10.1103/physrevaccelbeams.20.111003

4. Tarazona, D. A., et al. (2019). Dynamical simulations of the Muon Campus at Fermilab. Int. J. Mod. Phys. A 29, 2092004

5. Valetov, E. V. (2020). Toward the frontiers of particle physics with the Muon $g-2$ Experiment. https://doi.org/10.2172/1623356 